\title{
Prognostic Factors Affecting Surgical Outcomes in Squamous Cell Carcinoma of External Auditory Canal
}

\author{
Gi-Sung Nam · In Seok Moon · Ji Hyung Kim · Sung Huhn Kim · Jae Young Choi · Eun Jin Son \\ Department of Otorhinolaryngology, Yonsei University College of Medicine, Seoul, Korea
}

Objectives. Carcinomas of the external auditory canal (EAC) are rare, and management remains challenging. Previous studies seeking prognostic factors for EAC cancers included cancers other than carcinomas. In this study, we analyzed the treatment outcomes of, prognostic factors for, and survival rates associated with specifically squamous cell carcinoma (SCC) of the EAC.

Methods. A retrospective review of 26 consecutive patients diagnosed with SCCs of the EAC in a 10-year period was performed in terms of clinical presentation, stage, choice of surgical procedure, and adjunct therapy. Overall survival (OS) and recurrence-free survival (RFS) were calculated and univariate analysis of prognostic factors was performed.

Results. The median age of the 26 patients with SCCs of the EAC was 63 years (range, 40 to 72 years), and 16 males and 10 females were included. According to the modified University of Pittsburgh staging system, the T stages were T1 in 11,T2 in six, T3 in four, and T4 in five cases. The surgical procedures employed were wide excision in three cases, lateral temporal bone resection (LTBR) in 17, and extended LTBR in four, and subtotal temporal bone resection in two. Two patients underwent neoadjuvant chemotherapy, and two underwent adjuvant chemotherapy. One patient received preoperative radiation therapy, and eleven received postoperative radiation therapy. Of the possibly prognostic factors examined, advanced preoperative T stage and advanced overall stage were significant predictors of RFS, but not of OS.

Conclusion. The advanced $\mathrm{T}$ stage and overall stage were associated with decreased survival after surgical treatment in patients with SCC of the EAC, highlighting the importance of clinical vigilance and early detection.

Keywords. External Auditory Canal; Ear Neoplasms; Squamous Cell Carcinoma; Temporal Bone

\section{INTRODUCTION}

Carcinomas of the external auditory canal (EAC) are very rare, accounting for only $0.2 \%$ of all tumors of the head and neck [1]. Given such paucity, few epidemiological data are available and no consensus on management has emerged. Early diagnosis is

\footnotetext{
- Received September 29, 2017

Revised March 2, 2018

Accepted March 30, 2018

- Corresponding author: Eun Jin Son

Department of Otorhinolaryngology, Gangnam Severance Hospital, Yonsei University College of Medicine, 211 Eonju-ro, Gangnam-gu, Seoul 06273, Korea

$\mathrm{Tel}+82-2-2019-3460$, Fax +82-2-3463-4750,

E-mail: ejson@yuhs.ac
}

often difficult; biopsy is recommended in suspicious cases with EAC skin lesions [2]. Furthermore, treatment remains challenging due to the lack of reliable clinical and pathological prognostic factors, and the markedly diverse surgical and oncological treatment modalities available. Although no staging system for EAC carcinomas is currently recognized by either the Union for International Cancer Control or the American Joint Committee on Cancer, a comprehensive staging system developed by Arriaga et al. [3] and later modified by Moody et al. [1], namely the Pittsburgh Tumor Staging System, is most widely used $[4,5]$. In addition, advancements in radiological imaging including computed tomography (CT), magnetic resonance imaging (MRI), and positron emission tomography have provided clinicians with in-depth information that aids in preoperative staging and plan-

Copyright (C) 2018 by Korean Society of Otorhinolaryngology-Head and Neck Surgery.

This is an open-access article distributed under the terms of the Creative Commons Attribution Non-Commercial License (http://creativecommons.org/licenses/by-nc/4.0)

which permits unrestricted non-commercial use, distribution, and reproduction in any medium, provided the original work is properly cited. 
ning [4]. Treatment options include wide surgical resection, radiotherapy, chemotherapy, or combinations of these modalities. In operable cases, surgical approaches seek to achieve en bloc resection of the temporal bone using sleeve resection, lateral temporal bone resection (LTBR), or subtotal temporal bone resection (STBR), any of which can be combined with parotidectomy and/or neck dissection followed by (postoperative) radiotherapy [5-7]. However, the complexity of the anatomy and the relationship between the tumor with surrounding tissues within a limited space, render it difficult to attain safe resection margins [7]. The aim of this study was to report the treatment outcomes of patients with squamous cell carcinoma (SCC) of the EAC and to identify prognostic factors for survival in patients with this disease.

\section{MATERIALS AND METHODS}

This study included consecutive patients with histologically proven SCC of the EAC who underwent surgery between 2006 and 2016 at Severance Hospital and Gangnam Severance Hospital of Yonsei University Health System, Seoul, Korea. Retrospective review of the medical records was performed. The Institutional Review Board of Gangnam Severance Hospital reviewed and approved this study (IRB No. 3-2017-0219). As this was a retrospective study, no informed consent was required. Exclusion criteria were patients younger than 18 years of age or patients who had undergone treatment and recurrence occurred before initial presentation. Diagnostic work-up including physical examination, high-resolution CT and MRI, and preoperative biopsy was performed for all patients. The modified University of Pittsburgh staging system was used to assess the extents of primary and metastatic tumors for preoperative and pathological staging. Clinical presentation including, age, sex, date of diagnosis, sites of origin (bony and cartilaginous portions of EAC), and presence of ipsilateral facial nerve palsy or hearing loss were reviewed. The surgical treatment modalities included wide excision, LTBR, extended LTBR, or STBR, depending on the extent of the primary tumor. Total parotidectomy was indicated when

\section{HII G H L I G H T S}

- Early tumor stage squamous cell carcinomas of the external auditory canal had good prognosis and lower rate of recurrence compared to advanced cases.

- Among the prognostic factors analyzed, advanced tumor stages and neck node recurrence were associated with poor treatment outcomes.

- Our results highlight the importance of early diagnosis and surgical treatment for squamous cell carcinomas of the external auditory canal. the anterior growth of the tumor extended beyond the anterior wall of the EAC, whereas superficial parotidectomy was prophylactically performed on the intraparotid nodes in cases of T1 and T2 disease. Parotid involvement was suspected when imaging revealed erosion of the anterior wall of the auditory canal or an enhancing mass in the parotid area. During both LTBR and STBR, selective prophylactic neck dissection was performed on clinically negative necks, and modified radical neck dissection was performed on necks with clinically positive lymph nodes. Histologic grades and resection margin status were reviewed. Adjuvant postoperative chemoradiotherapy was prescribed for cases with positive or close resection margins, or clinically suspicious nodal involvement.

One patient was lost to follow up within 1 year after surgery, and remaining 25 patients were included for survival analysis. Overall survival (OS) was defined as the length of time from the start of treatment to the date of death from any cause or last follow-up, and recurrence-free survival (RFS), as the length of time after surgery for EC cancer until recurrence of cancer or last follow-up.

Statistical analyses were performed with the aid of SAS ver. 9.3 (SAS Institute Inc., Cary, NC, USA). Continuous variables were compared by the Mann-Whitney $U$-test, and categorical variables between two groups were compared using two-sided Fisher exact or the chi-square test. Survival rates were estimated using the Kaplan-Meier method and univariate survival analysis was completed using the log-rank test. A $P$-value $<0.05$ was considered statistically significant.

\section{RESULTS}

Between January 2006 and December 2016, 40 patients were newly diagnosed with EAC cancers and underwent surgical treatment. Twenty-six patients were diagnosed with SCC, eight with adenocystic carcinoma, five with adenocarcinoma, and one patient with basal cell carcinoma. We included 26 patients with confirmed SCC of the EAC for data analysis. One patient was lost to follow up within 1 year postoperatively, and was excluded from survival analysis. The patients included 16 males and 10 females, with a median age of 63 years (range, 40 to 72 years). The mean follow-up period was $2.9 \pm 1.9$ years. The site of involvement at presentation was the bony canal in nine patients $(33.3 \%)$, the cartilaginous canal in four $(14.8 \%)$, both bony and cartilaginous canals in eight (29.6\%), and unspecified in five patients $(18.5 \%)$ (Table 1$)$. The most common presenting symptom was otorrhea (nine patients, 33.3\%). Facial nerve palsy was present in three cases $(11.6 \%)$. Mass lesions in the EAC were visible otoscopically visible in most patients (24 cases, $92.3 \%$ ), except for two cases where only ulcerative lesions were evident in previously mastoidectomized ears.

Employing the University of Pittsburgh staging system, the T 
stages were $\mathrm{T} 1$ in 11 patients (42.3\%), T2 in six (23.1\%), T3 in four $(15.4 \%)$, and T4 in five $(19.2 \%)$. Preoperative regional lymph node involvement was evident in three patients $(12 \%)$; two patients $(7.7 \%)$ had stage N1 cancer and one $(3.8 \%)$ had stage N2 cancer. All of the patients underwent surgery; the surgical procedures included wide excision in three patients $(11.5 \%)$, LTBR in 17 (65.4\%), extended LTBR in four (14.8\%), and STBR in two $(7.1 \%)$. On pathological examination, tumor-free margins were achieved for 19 patients $(73.1 \%)$, but the other seven patients exhibited positive resection margins (26.9\%). A total of 20 patients $(76.9 \%)$ underwent ipsilateral parotidectomy: 12 (46.2\%) underwent superficial parotidectomy and eight (30.8\%) underwent total parotidectomy. Twelve patients (46.2\%) underwent ipsilateral neck dissection: selective dissection in nine $(34.6 \%)$, supraomohyoid dissection in two $(7.7 \%)$, and modified radical neck dissection in one (3.8\%). Additional surgical procedures were performed in three patients $(11.5 \%)$. One patient with a T1 tumor underwent LTBR with resection of the temporomandibular joint (TMJ), facial nerve resection, and cable graft anastomosis. LTBR combined with TMJ resection was performed in one patient with a T2 tumor. In one patient with a T4 tumor, STBR using the infratemporal fossa approach, TMJ resection, facial nerve resection, cable graft anastomosis, and partial resection of the dura were performed. The histological diagnoses were well-differentiated SCC in 19 patients (73.1\%),

Table 1. Baseline clinical characteristics of all patients with squamous cell carcinomas of EAC and recurred cases

\begin{tabular}{lcc}
\hline Variable & Total patients $(\mathrm{n}=26)$ & Recurrence $(\mathrm{n}=6)$ \\
\hline Age (yr), median (range) & $63(17-84)$ & $61(42-76)$ \\
Sex (male:female) & $16: 10$ & $4: 2$ \\
Location & & \\
Bony canal only & $9(33.3)$ & $1(16.7)$ \\
Cartilaginous canal only & $4(14.8)$ & $2(33.3)$ \\
Bony \& cartilaginous canal & $8(29.6)$ & $2(33.3)$ \\
Not specified & $5(18.5)$ & $1(16.7)$ \\
Histology & & \\
Well differentiated & $16(61.5)$ & $1(16.7)$ \\
Moderately differentiated & $6(23.1)$ & $1(16.7)$ \\
Poorly differentiated & $1(3.8)$ & $3(50.0)$ \\
Not specified & $3(11.5)$ & $1(16.7)$ \\
T stage (modified Pittsburg) & & \\
T1 & $10(37.0)$ & $1(16.7)$ \\
T2 & $8(29.6)$ & $3(50.0)$ \\
T3 & $4(14.8)$ & 0 \\
T4 & $4(14.8)$ & $2(33.3)$ \\
N stage (modified Pittsburg) & & \\
N1 & $24(92.3)$ & $6(100)$ \\
N2 & $1(3.8)$ & 0 \\
N3 & $1(3.8)$ & 0 \\
N4 & 0 & 0 \\
\hline & &
\end{tabular}

Values are presented as number or number (\%) unless otherwise indicated. EAC, external auditory canal. moderately differentiated SCC in six $(23.1 \%)$, and poorly differentiated SCC in one (3.8\%). Grading reports for three cases were lacking. Postoperative adjuvant radiotherapy was prescribed for 12 patients (46\%). One patient staged as T4N0M0 underwent neoadjuvant chemoradiotherapy before surgery (extended LTBR) and two patients with positive resection margins received adjuvant chemotherapy. Treatment modalities according to the tumor stages are shown in Table 2.

Locoregional recurrence developed in six patients (23\%) at a median follow-up time of 3.0 years (range, 1.1 to 7.9 years): three patients exhibited local failure, two had neck node recurrences, and one had both local and neck node recurrences (Table 1). The recurrence rates were significantly higher in those of more advanced tumor stages: no pT1 patient experienced recurrence, but four, none, and two pT2, pT3, and pT4 cases, respectively, experienced recurrences $(P=0.014)$. Most recurrences (five

Table 2. Tumor stage-related treatment modalities $(n=26)$

\begin{tabular}{lcccc}
\hline Variable & $\begin{array}{c}\mathrm{T} 1 \\
(\mathrm{n}=10)\end{array}$ & $\begin{array}{c}\mathrm{T} 2 \\
(\mathrm{n}=8)\end{array}$ & $\begin{array}{c}\mathrm{T} 3 \\
(\mathrm{n}=4)\end{array}$ & $\begin{array}{c}\mathrm{T} 4 \\
(\mathrm{n}=4)\end{array}$ \\
\hline $\begin{array}{l}\text { Type of surgery } \\
\quad \text { Wide excision }\end{array}$ & 2 & 0 & 1 & 0 \\
$\quad$ Lateral temporal bone resection & 7 & 8 & 1 & 1 \\
$\quad \begin{array}{l}\text { Extended lateral temporal bone resection } \\
\quad 1\end{array}$ & 0 & 2 & 1 \\
$\quad \begin{array}{l}\text { Subtotal temporal bone resection } \\
\text { Additional surgery }\end{array}$ & 0 & 0 & 0 & 2 \\
$\quad \begin{array}{l}\text { Subtotal parotidectomy } \\
\text { Total parotidectomy }\end{array}$ & 6 & 1 & 4 & 1 \\
$\quad$ Neck dissection & 1 & 4 & 0 & 3 \\
Adjuvant treatment & 4 & 3 & 2 & 3 \\
$\quad \begin{array}{l}\text { Chemotherapy } \\
\text { Radiotherapy }\end{array}$ & 0 & 1 & 1 & 1 \\
\hline
\end{tabular}

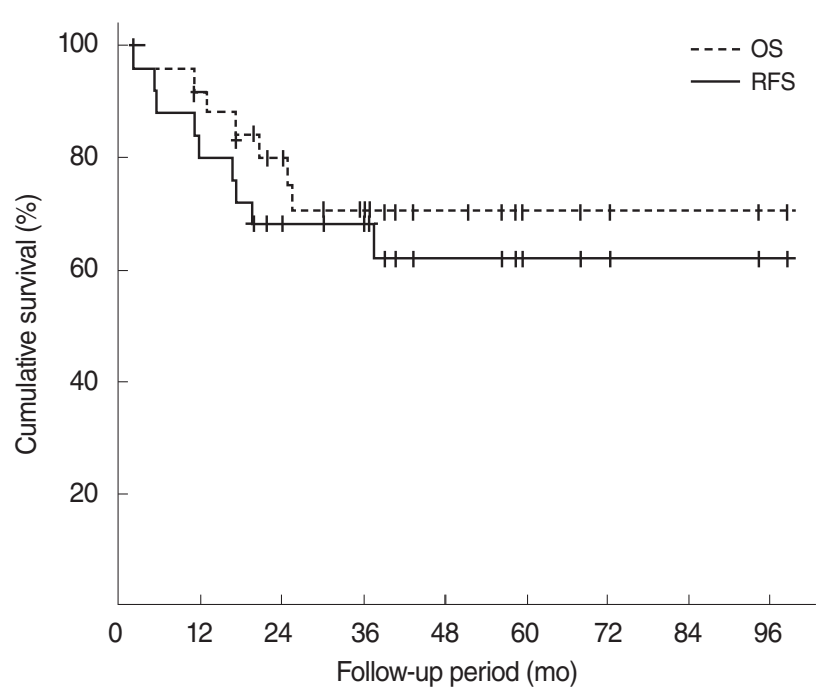

Fig. 1. Kaplan-Meier curves for overall survival (OS) and recurrencefree survival (RFS) in patients with squamous cell carcinoma of the external auditory canal. The 5-year OS was $70.4 \%$ and RFS was $61.8 \%$. 
Table 3. Univariate analysis of prognostic factors for OS and RFS in patients with squamous cell carcinoma of EAC $(n=25)$

\begin{tabular}{|c|c|c|c|c|}
\hline \multirow{2}{*}{ Variable } & \multicolumn{2}{|c|}{ OS } & \multicolumn{2}{|c|}{ RFS } \\
\hline & $\operatorname{HR}(95 \% \mathrm{Cl})$ & $P$-value & $\mathrm{HR}(95 \% \mathrm{Cl})$ & $P$-value \\
\hline Age & $0.98(0.92-1.05)$ & 0.573 & $0.99(0.94-1.06)$ & 0.903 \\
\hline Sex & $1.17(0.26-5.24)$ & 0.838 & $1.11(0.30-4.17)$ & 0.880 \\
\hline Facial nerve palsy, present & $1.29(0.06-27.87)$ & 0.869 & $0.95(0.05-19.42)$ & 0.976 \\
\hline Hearing loss, present & $1.03(0.20-5.30)$ & 0.974 & $0.67(0.14-3.21)$ & 0.612 \\
\hline Advanced preoperative T stage (T2, T3, T4) & $13.74(0.64-296.97)$ & 0.095 & $8.57(1.05-70.20)$ & 0.045 \\
\hline Advanced preoperative N stage (N1, N2, N3) & $1.45(0.17-12.07)$ & 0.733 & $1.15(0.14-9.92)$ & 0.896 \\
\hline Advanced preoperative stage (II, III, IV) & $13.74(0.64-296.97)$ & 0.095 & $8.57(1.05-70.20)$ & 0.045 \\
\hline Types of surgery (ETBR, STBR) & $2.23(0.10-47.68)$ & 0.608 & $3.00(0.15-60.49)$ & 0.474 \\
\hline Resection margin (positive for malignancy) & $4.89(0.94-25.48)$ & 0.060 & $3.14(0.81-12.19)$ & 0.099 \\
\hline Histology grade (moderately-, poorly-differentiated) & $0.15(0.02-1.54)$ & 0.110 & $0.28(0.03-2.29)$ & 0.253 \\
\hline Advanced pathologic T stage (T2, T3, T4) & $10.28(0.48-220.21)$ & 0.136 & $15.16(0.75-307.02)$ & 0.077 \\
\hline Advanced pathologic N stage (N1, N2, N3) & $6.11(0.62-59.92)$ & 0.120 & $2.61(0.32-21.35)$ & 0.371 \\
\hline Advanced final stage (II, III, IV) & $10.28(0.48-220.21)$ & 0.136 & $15.16(0.75-307.02)$ & 0.077 \\
\hline Chemotherapy & $0.22(0.04-1.26)$ & 0.089 & $0.41(0.08-2.04)$ & 0.275 \\
\hline Radiotherapy & $2.27(0.20-225.59)$ & 0.508 & $2.58(0.23-29.09)$ & 0.444 \\
\hline
\end{tabular}

OS, overall survival; RFS, recurrence-free survival; EAC, external auditory canal; HR, hazard ratio; Cl, confidence interval; ETBR, extended temporal bone resection; STBR, subtotal temporal bone resection.

of six, $83.3 \%$ ) were treated with adjuvant radiotherapy, and one patient with a pT2 tumor underwent chemotherapy. During follow-up, four of the recurrent patients died of disease-related conditions.

Survival outcomes were analyzed for the 25 patients with follow-up period of at least 1 year. The median follow-up period was 36.3 months (range, 2.2 to 98.6 months). The 5 -year OS was $70.4 \%$ and RFS was $61.8 \%$ (Fig. 1). When various predictive factors of the OS and RFS were analyzed using univariate analysis (Table 3), preoperative T stage (T2,T3, and T4 compared to T1) and preoperative overall stage (stages II, III, IV compared to stage I) were significantly associated with RFS, but not OS $(P<0.05)$. Other variables such as age $(>60$ years vs. $\leq 60$ years), sex (male vs. female), presence of facial palsy or hearing loss, preoperative N stage (N1, N2, N3 vs. N0), types of surgery (ETBR and STBR vs. others), resection margins (positive vs. negative for malignancy), histologic grade (moderately or poorly differentiated vs. well-differentiated), pathologic T stage (T2, T3, $\mathrm{T} 4$ vs. T1), pathologic $\mathrm{N}$ stage (N1, N2, N3 vs. N0), final stage (II, III, IV vs. I), and addition of chemotherapy/radiotherapy did not show significant associated with survival outcomes.

\section{DISCUSSION}

In general, EAC cancer has a poor prognosis, and management remains challenging. Efforts have been made to identify prognostic factors that can improve treatment protocols. Several disease-related factors such as tumor stage, a poorly differentiated cell type, neck lymph node involvement, and facial paralysis indicate poor prognosis [8-11]. In terms of primary tumors, some studies have suggested that extensive local invasion, middle-ear involvement, facial nerve palsy, positive surgical margins, and cerebral involvement are associated with poor prognosis, whereas others have reported that cerebral invasion is not a negative prognostic factor [6,11-14]. Ensuring that the margin is tumorfree after surgical resection is important. Essig et al. [15] reported a DFS rate of $81 \%$ at 2 years in patients with clear margins, in contrast to a DFS rate of $45 \%$ in patients with positive margins. Moody et al. [1] also reported that positive histological margins were associated with reduced survival at 2 years (32\%) compared to that of patients with clear margins $(75 \%)$ [1]. We found that the 2-year OS rate of patients with positive margins was poorer than that of those with clear margins $(57.1 \%$ vs. $81.3 \%$ ). However, it should be noted that SCCs of the EAC are associated with a significant recurrence rate even in patients for whom postoperative pathological examinations reveal clear margins [16]. Nonetheless, the surgeon should consider wide en bloc resection to create oncologically safe margins, particularly of bone tissue, during the preoperative work-up [17].

A role for parotidectomy in treatment of advanced EAC cancer has been reported in many studies. We previously found that parotid invasion by SCC was only evident in advanced cases, and could be detected during preoperative evaluation [18]. In our study, although no patient exhibited lymph node involvement on postoperative pathological examination, three patients developed nodal recurrences during follow-up. This affords a rationale for prophylactic parotidectomy and selective neck dissection when treating SCC in the EAC. Ihler et al. [5] reported that EAC cancer patients who did not undergo parotidectomy (with or without neck dissection) had a mean survival time shorter than the average of all EAC cancer patients. Although a consistent management strategy for the parotid gland has not emerged, recent studies have suggested that at least superficial 


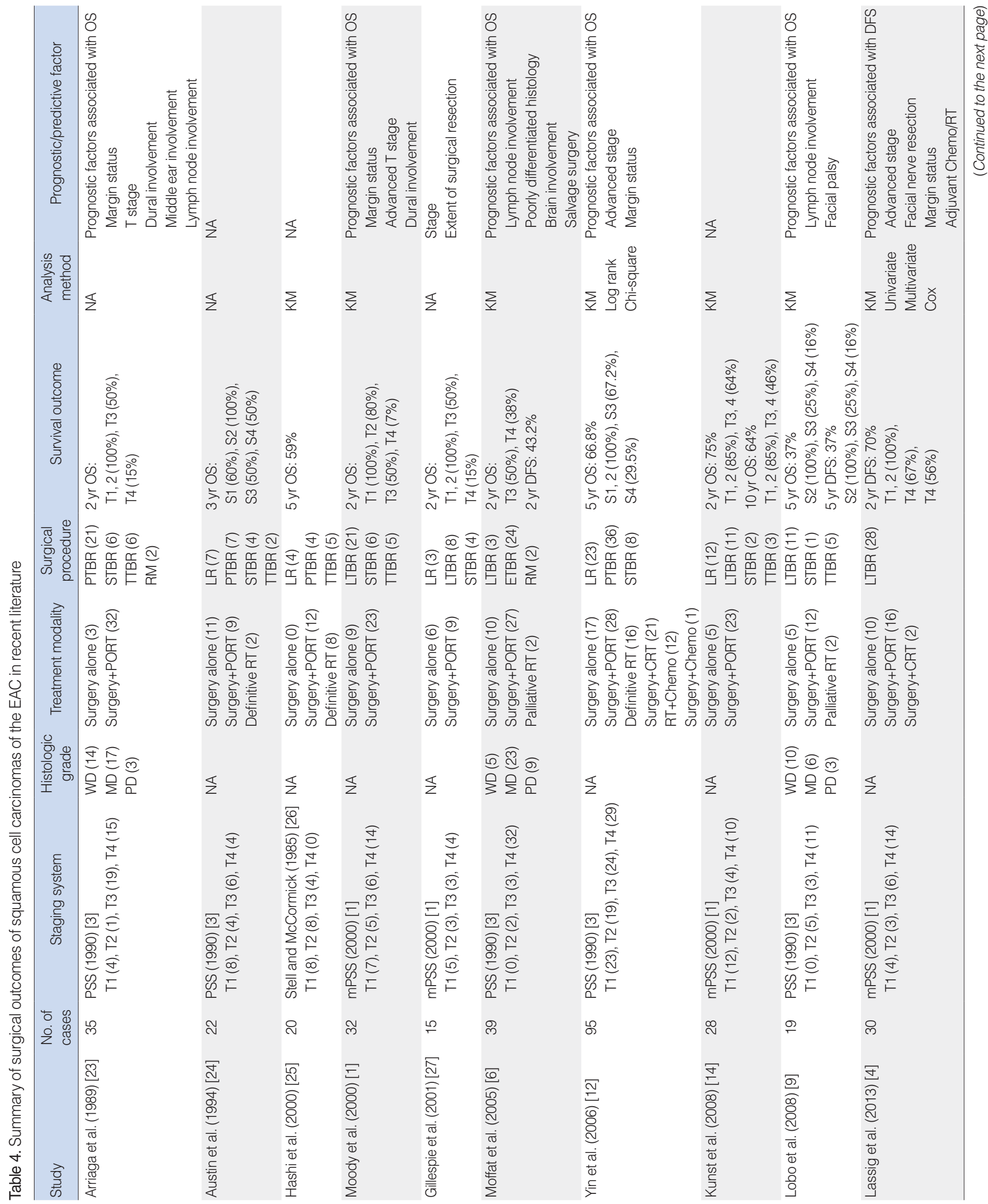




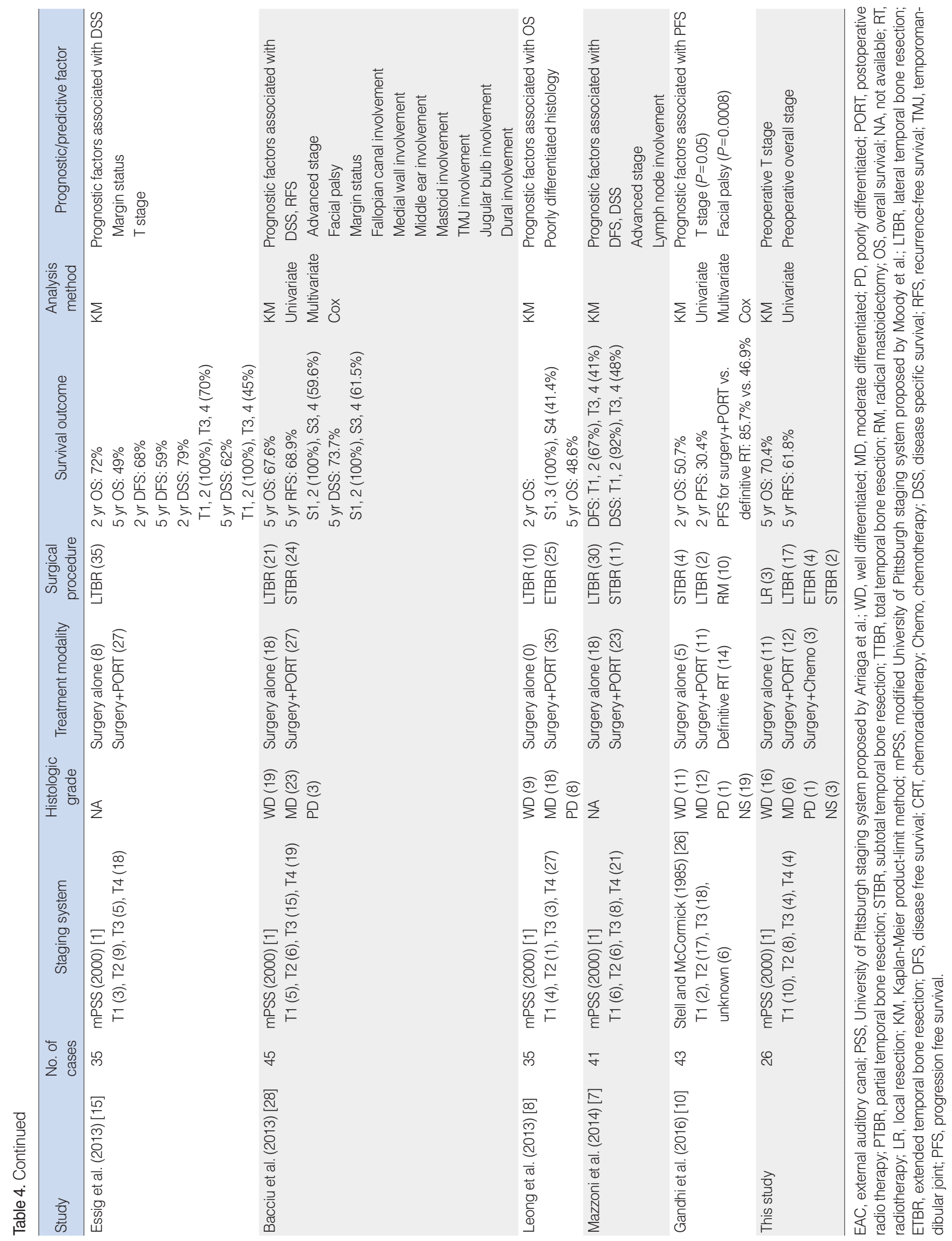


parotidectomy is required even when clinical evidence of lymphatic involvement is absent $[4,6,8,17,19]$. Mazzoni et al. [7] advocated total parotidectomy when the extent of anterior growth of the SCC extended beyond the anterior wall of the EAC, and prophylactic superficial parotidectomy for patients with $\mathrm{T} 1$ or $\mathrm{T} 2$ disease. The cited authors performed modified radical neck dissection to treat clinically positive lymph nodes, and selective prophylactic neck dissection in patients with clinically negative necks who underwent LTBR, or STBR. Total parotidectomy was recommended for patients with advanced SCCs [20].

Although SCC is the most common histological type of temporal bone neoplasm, other carcinoma subtypes include basal cell carcinoma, adenocarcinoma, adenoid cystic carcinoma, mucoepidermoid carcinoma, melanoma, and sarcoma. In addition, some studies included parotid gland tumors invading the EAC and SCCs arising from the auricle as EAC cancers. As histological subtype may be a confounding factor, we only collected data from histologically confirmed SCCs. SCCs of the EAC are associated with poorer prognoses than other pathologies. Treatment outcomes have improved over the past several decades because of advances in imaging modalities and microsurgical and chemoradiotherapeutic techniques. In a review of the literature, Prasad et al. [21] reported the 2-year survival rates of patients with temporal bone cancers by grade: T1 $(48 \%-100 \%)$, T2 $(28 \%-$ $100 \%)$, T3 (17\%-100\%), and T4 (14.3\%-54.0\%). The authors attributed the wide ranges to difficulties in collecting data on a rare disease and the use of different treatment modalities [21]. In our study, the OS rate was $75.4 \%$ and the 5 -year survival rates were as follows: $\mathrm{T} 1$ (90.0\%), T2 $(44.0 \%), \mathrm{T} 3(75.0 \%)$, and T4 $(40.0 \%)$. In another study seeking predictors of survival/recurrence after temporal bone resection, the relevant predictors were the status of the surgical margin, metastatic lymph nodes in the neck or parotid, and parotid invasion [22]. Our results indicated that only $\mathrm{T}$ stage, which is associated with the difficulty achieving a safe margin because of the complex anatomy and surrounding vital tissues, affected OS and DFS. The survival rates of patients with SCCs of the EAC who undergo surgery at early stages are reportedly favorable, highlighting the importance of clinical suspicion and early diagnosis. The results of our study have been summarized along with the results of contemporary series in Table 4 [1,3,4,6-10,12,14,15,23-28].

The greatest limitations of our study were the small number of patients, particularly those of tumor stages $\mathrm{T} 3$ and $\mathrm{T} 4$, and the limited follow-up period. Detailed longer-term follow-up of larger cohorts of patients with all stages of disease would be invaluable to strengthen the statistical analysis. In addition, more comprehensive information on prognostic factors would provide much-needed evidence that would allow the treatment guidelines for SCC of the EAC to be refined.

In conclusion, we identified that preoperatively advanced tumor stages are related to decreased survival outcomes in pa- tients with SCC of the EAC who underwent surgical treatment. Our results highlight the importance of clinical vigilance and early detection of EAC lesions. However, further studies with comprehensive evaluation for clinical and surgical prognostic factors would provide better insight to surgical outcomes for SCC of the EAC.

\section{CONFLICT OF INTEREST}

No potential conflict of interest relevant to this article was reported.

\section{ACKNOWLEDGMENTS}

We would like to thank Dr. Hye Sun Lee and Ms. Sinae Kim of the Biostatistics Collaboration Unit, Yonsei University College of Medicine for their contributions to this work and their constructive collaboration.

This research was supported by the Basic Science Research Program of the National Research Foundation of Korea (NRF), funded by the Ministry of Education, Science and Technology (Grant No. 2016R1A2B1012521 to EJS), Republic of Korea, and by a faculty research grant from Yonsei University College of Medicine (6-2016-0040), Seoul, Korea.

\section{REFERENCES}

1. Moody SA, Hirsch BE, Myers EN. Squamous cell carcinoma of the external auditory canal: an evaluation of a staging system. Am J Otol. 2000 Jul;21(4):582-8.

2. Zhang T, Dai C, Wang Z.The misdiagnosis of external auditory canal carcinoma. Eur Arch Otorhinolaryngol. 2013 May;270(5):1607-13.

3. Arriaga M, Curtin H, Takahashi H, Hirsch BE, Kamerer DB. Staging proposal for external auditory meatus carcinoma based on preoperative clinical examination and computed tomography findings. Ann Otol Rhinol Laryngol. 1990 Sep;99(9 Pt 1):714-21.

4. Lassig AA, Spector ME, Soliman S, El-Kashlan HK. Squamous cell carcinoma involving the temporal bone: lateral temporal bone resection as primary intervention. Otol Neurotol. 2013 Jan;34(1):14150.

5. Ihler F, Koopmann M, Weiss BG, Droge LH, Durisin M, Christiansen $\mathrm{H}$, et al. Surgical margins and oncologic results after carcinoma of the external auditory canal. Laryngoscope. 2015 Sep;125(9):210712.

6. Moffat DA, Wagstaff SA, Hardy DG. The outcome of radical surgery and postoperative radiotherapy for squamous carcinoma of the temporal bone. Laryngoscope. 2005 Feb;115(2):341-7.

7. Mazzoni A, Danesi G, Zanoletti E. Primary squamous cell carcinoma of the external auditory canal: surgical treatment and long-term outcomes. Acta Otorhinolaryngol Ital. 2014 Apr;34(2):129-37.

8. Leong SC, Youssef A, Lesser TH. Squamous cell carcinoma of the temporal bone: outcomes of radical surgery and postoperative radiotherapy. Laryngoscope. 2013 Oct;123(10):2442-8.

9. Lobo D, Llorente JL, Suarez C. Squamous cell carcinoma of the ex- 
ternal auditory canal. Skull Base. 2008 May;18(3):167-72.

10. Gandhi AK, Roy S, Biswas A, Raza MW, Saxena T, Bhasker S, et al. Treatment of squamous cell carcinoma of external auditory canal: a tertiary cancer centre experience. Auris Nasus Larynx. 2016 Feb; 43(1):45-9.

11. Ouaz K, Robier A, Lescanne E, Bobillier C, Moriniere S, Bakhos D. Cancer of the external auditory canal. Eur Ann Otorhinolaryngol Head Neck Dis. 2013 Sep;130(4):175-82.

12. Yin M, Ishikawa K, Honda K, Arakawa T, Harabuchi Y, Nagabashi T, et al. Analysis of 95 cases of squamous cell carcinoma of the external and middle ear. Auris Nasus Larynx. 2006 Sep;33(3):251-7.

13. Hosokawa S, Mizuta K, Takahashi G, Okamura J, Takizawa Y, Hosokawa $\mathrm{K}$, et al. Carcinoma of the external auditory canal: histological and treatment groups. B-ENT. 2014;10(4):259-64.

14. Kunst H, Lavieille JP, Marres H. Squamous cell carcinoma of the temporal bone: results and management. Otol Neurotol. 2008 Jun; 29(4):549-52.

15. Essig GF, Kitipornchai L, Adams F, Zarate D, Gandhi M, Porceddu S, et al. Lateral temporal bone resection in advanced cutaneous squamous cell carcinoma: report of 35 patients. J Neurol Surg B Skull Base. 2013 Feb;74(1):54-9.

16. Marioni G, Martini A, Favaretto N, Franchella S, Cappellesso R, Marino $\mathrm{F}$, et al. Temporal bone carcinoma: a first glance beyond the conventional clinical and pathological prognostic factors. Eur Arch Otorhinolaryngol. 2016 Oct;273(10):2903-10.

17. Zanoletti E, Marioni G, Franchella S, Lovato A, Giacomelli L, Martini A, et al. Recurrent squamous cell carcinoma of the temporal bone: critical analysis of cases with a poor prognosis. Am J Otolaryngol. 2015 May-Jun;36(3):352-5.

18. Choi JY, Choi EC, Lee HK, Yoo JB, Kim SG, LeeWS. Mode of parotid involvement in external auditory canal carcinoma. J Laryngol Otol. 2003 Dec;117(12):951-4.

19. Xie B, Zhang T, Dai C. Survival outcomes of patients with temporal bone squamous cell carcinoma with different invasion patterns. Head Neck. 2015 Feb;37(2):188-96.

20. Homer JJ, Lesser T, Moffat D, Slevin N, Price R, Blackburn T. Management of lateral skull base cancer: United Kingdom National Multidisciplinary Guidelines. J Laryngol Otol. 2016 May;130(S2): S119-24.

21. Prasad SC, D’Orazio F, Medina M, Bacciu A, Sanna M. State of the art in temporal bone malignancies. Curr Opin Otolaryngol Head Neck Surg. 2014 Apr;22(2):154-65.

22. Morris LG, Mehra S, Shah JP, Bilsky MH, Selesnick SH, Kraus DH. Predictors of survival and recurrence after temporal bone resection for cancer. Head Neck. 2012 Sep;34(9):1231-9.

23. Arriaga M, Hirsch BE, Kamerer DB, Myers EN. Squamous cell carcinoma of the external auditory meatus (canal). Otolaryngol Head Neck Surg. 1989 Sep;101(3):330-7.

24. Austin JR, Stewart KL, Fawzi N. Squamous cell carcinoma of the external auditory canal: therapeutic prognosis based on a proposed staging system. Arch Otolaryngol Head Neck Surg. 1994 Nov;120(11):122832.

25. Hashi N, Shirato H, Omatsu T, Kagei K, Nishioka T, Hashimoto S, et al. The role of radiotherapy in treating squamous cell carcinoma of the external auditory canal, especially in early stages of disease. Radiother Oncol. 2000 Aug;56(2):221-5.

26. Stell PM, McCormick MS. Carcinoma of the external auditory meatus and middle ear: prognostic factors and a suggested staging system. J Laryngol Otol. 1985 Sep;99(9):847-50.

27. Gillespie MB, Francis HW, Chee N, Eisele DW. Squamous cell carcinoma of the temporal bone: a radiographic-pathologic correlation. Arch Otolaryngol Head Neck Surg. 2001 Jul;127(7):803-7.

28. Bacciu A, Clemente IA, Piccirillo E, Ferrari S, Sanna M. Guidelines for treating temporal bone carcinoma based on long-term outcomes. Otol Neurotol. 2013 Jul;34(5):898-907. 